delay execution of the later one. But dependency would still be maintained if a control pathway was included to ensure that the later event would not be undertaken unless the earlier event was completed.

Hartwell and Weinert ${ }^{1}$ analysed such control pathways and have proposed the term checkpoint to describe the situation in which an early event is kept under surveillance; if the event is not properly finished, a signal then blocks a later, dependent event. Checkpoints thus enable the cell to monitor progression through the sequence of essential stages of the cell cycle. Unlike the substrateproduct dependency, it should be possible to uncouple two sequential events artificially. For example, mutations or chemical treatments that alter one of the steps in the checkpoint control might allow the later event to proceed without completion of the earlier one.

Hoyt et al. ${ }^{2}$ and Li and Murray ${ }^{3}$ have now proposed that a checkpoint control monitors spindle function during mitosis. A microtubular spindle is required for proper segregation of the chromosomes, and impaired spindle function leads to chromosome missegregation. If the spindle is disrupted with microtubular poisons, such as benomyl or nocodazole, progression through mitosis is blocked. The authors have established that a checkpoint control is involved in this dependency of mitosis on spindle function by isolating mutants in budding yeast that complete mitosis and cell division even when the spindle is not functioning properly.

Two different but related procedures for mutant isolation were used. Hoyt et $a l .{ }^{2}$ treated cells with enough benomyl to knock out spindle function completely. They then screened for mutants that managed to recover after removal of benomyl, arguing that any mutant cells unable to detect or respond to the disrupted spindle function would proceed through mitosis into cell division. Cells dividing in these circumstances would make a poor recovery after benomyl withdrawal and would mostly die because of chromosome missegregation. Three mutants were isolated that continued to divide in the presence of benomyl. The mutants defined two genes, $B U B 1$ and 2 (budding uninhibited by benzimadazole). A third gene, $B U B 3$, identified initially as a multicopy suppressor of $B U B I$, also generated a similar mutant phenotype when deleted.

$\mathrm{Li}$ and Murray ${ }^{3}$ treated cells with a low level of benomyl, which did not completely disrupt spindle function but did slow down spindle assembly. Mutants were isolated that proceeded into cell division at a normal rate during this treatment. These died as a result of chromosome missegregation due to in- complete spindle assembly. Five mutants isolated in this way defined three genes, $M A D 1,2$ and 3 (mitotic arrest deficient). Because of strain differences, allelism testing between the two sets of mutants is difficult, but the mutants define between four and six genes that might influence the checkpoint control. The fact that only a few mutants have identified so many genes suggests that either the control system is complex and involves many gene products, or the control is indirectly affected by other gene products whose function is primarily concerned with other processes.

These analyses indicate that the mutated genes influence a specific control that checks for proper spindle function before mitosis is allowed to proceed. That spindle malfunction is a checkpoint is demonstrated by the fact that cell cycle mutants leading to other defects in mitotic progression do not complete mitosis and cell division if combined with bub mutations. Also, the drop in cell viability of mad mutants treated with benomyl occurs only when the mutant cells complete mitosis and is totally suppressed if mitosis cannot take place because of an earlier block in the cell cycle. As expected, treatment of the mutants with benomyl massively increases chromosome losses, and the stability of chromosomes is reduced even in the absence of benomyl. This second result suggests that these gene products do indeed contribute to the fidelity of chromosome transmission during the normal cell cycle.

What is the mechanism by which this checkpoint control prevents further progression through mitosis? One possibility is that it operates by influencing inactivation of the p34 mitotic kinase which brings about mitosis ${ }^{4}$. Cells with persistently high p34 kinase activity cannot proceed from mitosis into interphase until this activity drops ${ }^{5,6}$. When the mitotic spindle is damaged, either by mutants or microtubular poisons, cells arrest with their p34 kinase activity still raised. But when the spindle is disrupted in the bub or mad mutants, the p34 kinase activity is not so high and thereafter slowly declines. Perhaps the normal role of the $B U B$ and $M A D$ gene products is to maintain increased p34 kinase activity should the spindle malfunction, so preventing exit from mitosis until the problem is corrected. In the $b u b$ and mad mutants, the p34 kinase activity cannot be kept up and so cells gradually exit from mitosis and enter interphase.

Little is yet known about the molecular basis of $B U B$ and $M A D$ gene function. In principle, they could influence any steps in the checkpoint control pathway, including the surveillance of spindle function, the signal generated when the spindle malfunctions, and the response

\section{Hum drum}

WILL discothèques of the future echo to the beat of the fractal drum, described in Physical Review Letters (67, 2974 2977; 1991) by B. Sapoval and colleagues? The drum was designed to test the notion that fractal borders may influence the behaviour of waves, particularly the damping of waves. The drum rim, rather than being the familiar circle, is a sort of cruciform snowflake. To find the drum's resonant frequencies, Sapoval et al. excited the drumhead by humming at it with sine tones from a loud speaker. A laser beam skimming over the surface revealed any vibrations. One surprise was to find resonant oscillatory modes that involved only one limb of the 'cross', leaving the rest of the drumhead unmoved. The authors believe the confinement to be a result of competition between wave damping and propagation, the wave's energy being soaked up in the minutiae of the drum's fractal border before it is transmitted through the constricted neck that joins each limb to the main body of the cross.

\section{Acting on actin}

CALDESMON is part of a calcium-controlled regulatory system that acts on the thin filaments of smooth muscle. It binds to $\mathrm{F}$-actin, and a binding site close to the $\mathrm{N}$ terminus of the actin has been found. Now a second site at the $C$ terminus is reported in two separate papers by $\mathrm{R}$. Crosbie et al. and P. Graceffa and A. Jancso (J. biol. Chem. 266, 2000120006 \& 20205-20210; 1991). The existence of binding sites for parts of the myosin head at both the $\mathrm{N}$ and $\mathrm{C}$ termini of the actin subunits suggests that inhibition of contraction may operate by simple steric obstruction.

\section{Beer break}

BEER glasses made from toughened glass are hopeless as weapons, say J. P Shepherd et al. in the British Medical Journal (303, 1330; 1991). Assaults with fractured pint glasses in the less salubrious of Britain's pubs leave 70 per cent of the victims with permanent scars and cost the state more than $£ 1$ million a year in compensation. Shepherd and colleagues tested the impact resistance of several brands of pint glass, both the tankard and the straight variety, and found that toughened straight glasses - even when slightly damaged or scratched - can withstand physical insults far better than the usual annealed straight glasses and tankards; and even when they break, they shatter into myriads of tiny blunt-edged cubes. As well as being more durable, toughened glasses are actually cheaper and even the most sober of patrons cannot tell the difference. The researchers recommend that magistrates make their use a condition of licensing. 